\title{
Cuidado em saúde mental e atenção primária em saúde como campo formador para a enfermagem
}

Bárbara Jéssica de Melo Cezar Dias ${ }^{1}$

(D) https://orcid.org/0000-0001-7486-3317

Fernanda Martinez de Oliveira Resende ${ }^{1}$

(1D) https://orcid.org/0000-0001-7167-4860

Giulia Ribeiro Schettino Regne ${ }^{1}$

(D) https://orcid.org/0000-0002-7287-8635

Amanda Márcia dos Santos Reinaldo ${ }^{1}$

(D) https://orcid.org/0000-0003-0283-2313

Maria Odete Pereira ${ }^{1}$

(D) https://orcid.org/0000-0002-9418-2524

Marcus Luciano de Oliveira Tavares ${ }^{1}$

(DD https://orcid.org/0000-0002-8598-7603

Universidade Federal de Minas Gerais, Belo Horizonte, MG, Brasil.
Objetivo: apresentar o olhar de duas acadêmicas do último ano do curso de enfermagem de uma instituição federal de ensino superior sobre um serviço da atenção primária em saúde em relação ao cuidado em rede à pessoa em sofrimento mental. Método: estudo descritivo do tipo relato de experiência apresentado por meio de narrativa, que foi construída a partir do registro em diários de campo. Resultados: observa-se a não articulação entre teoria e prática no atendimento em rede à pessoa em sofrimento mental; considera-se a necessidade de observar o cenário de prática articulado à realidade onde o serviço está inserido, apontando-se a necessidade de avaliação da formação do futuro enfermeiro nesse sentido. Conclusão: o ponto de vista do aluno contribui para pensar a prática docente no ensino da saúde mental enquanto componente que perpassa a saúde global.

Descritores: Saúde Mental; Atenção Primária em Saúde; Enfermagem; Educação.

\section{Como citar este artigo}

Dias BJMC, Resende FMO, Regne GRS, Reinaldo AMS, Pereira MO, Tavares MLO. Mental health care and primary health care as a training field for nurses. SMAD, Rev Eletrônica Saúde Mental Álcool Drog. 2020;16(2):1-8. doi: https://dx.doi.org/10.11606/issn.1806-6976.smad.2020.153900 


\section{Mental health care and primary health care as a training field for nurses}

Objective: to present the view of two students from the last year of an undergraduate nursing program at a federal higher-education institution concerning a primary health care service and the network care provided to individuals with mental suffering. Method: this is a descriptive experience report presented through a narrative, which was produced from records on field diaries. Results: there is a lack of articulation between theory and practice in the network care provided to individuals with mental suffering; the need to observe the practice scenario in articulation with the reality where the service operates is considered. Conclusion: the students' point of view contributes to the reflection on the teaching practice in mental health as a component that permeates global health.

Descriptors: Mental Health; Primary Health Care; Nursing; Education.

\section{Atención de salud mental y atención primaria de salud como campo de capacitación en enfermería}

Objetivo: presentar la opinión de dos estudiantes del último año del Curso de Enfermería de una Institución Federal de Educación Superior sobre un servicio de atención primaria de salud en relación con la atención de la persona con problemas mentales en una red. Método: un estudio descriptivo del informe de experiencia tipo presentado a través de la narrativa, que se construyó a partir del registro en diarios de campo. Resultados: existe una falta de articulación entre la teoría y la práctica en la observación de la atención en red de la persona con angustia mental; Se considera la necesidad de observar el escenario de práctica articulada la realidad donde se inserta el servicio, la necesidad de evaluar la formación de futuras enfermeras en este sentido. Conclusión: el punto de vista del alumno contribuye a pensar que la práctica docente en la enseñanza de la salud mental es un componente que impregna la salud global.

Descriptores: Salud Mental; Atención Primaria de Salud; Enfermería; Educación. 


\section{Introdução}

As ideias de movimento, projeto, mudança, construção e reconstrução, ocupação de espaços/ territórios e práticas de inclusão perpassam a reforma psiquiátrica no Brasil e no mundo. A participação social se fez e faz necessária nesse movimento político em que se redirecionou a atenção ao sofrimento mental, em consonância com os princípios do Sistema Único de Saúde (SUS), a reforma sanitária brasileira e a redemocratização do país(1-2).

Para sustentar essa mudança no modo de ver e viver a loucura foi necessário pensar a sociedade e a comunidade como espaço social da pessoa em sofrimento mental, sendo que esse sujeito não pode ser cuidado fora desse espaço, sob o risco de institucionalização de pessoas e profissionais com suas práticas na clínica do sofrimento mental.

No lugar dos serviços substitutivos ao hospital psiquiátrico e sua lógica de trabalho, foi criada a Rede de Atenção Psicossocial, que deve trabalhar sob a lógica da atenção do sujeito em rede, considerando os princípios da reabilitação psicossocial e o reconhecimento do sujeito enquanto detentor de direitos e autonomia(1).

A reforma psiquiátrica brasileira promoveu mudanças e gerou avanços nas políticas públicas no contexto social e do Sistema Único de Saúde. Em que pese às críticas ao modelo e os retrocessos, observados nos últimos anos, em detrimento das questões técnicas que sustentam um modelo de atenção psicossocial, podemos considerar que pensar saúde e sofrimento mental sem considerar o sujeito de direito garantido pela constituição brasileira e sua inserção no território que habita é um retrocesso por si só(2).

É no território que habita a identidade do sujeito e vice-versa. A atenção à pessoa em sofrimento mental, quando se dá no território, opera a partir das necessidades de saúde do sujeito, no que ele tem de comum à comunidade e singular à sua vida. É nesse lugar que se produz saúde, na casa, na rua, na escola, no centro comunitário, no projeto social, nas agências religiosas e também na atenção primária em saúde(3).

Nesse sentido, utilizar o serviço de saúde diz sobre a relação do sujeito com a rede de cuidados em que ele se insere, com os trabalhadores dos serviços e com a oferta de serviços, considerando os determinantes sociais em saúde e em saúde mental(4-5).

O serviço de saúde trabalha com a demanda que Ihe é apresentada e a partir da lógica da referência e contrarreferência, do cuidado no território, dos princípios que sustentam e fazem o SUS acontecer nos seus 30 anos, entre outros fatores, permitindo que o sofrimento mental, independentemente de sua gravidade, passasse a fazer parte do cotidiano das unidades de saúde que compõem a rede de atenção em saúde ${ }^{(6-7)}$.
O cuidado da pessoa em sofrimento mental na atenção primária à saúde tem potencialidades e fragilidades que são discutidas amplamente pelos atores sociais dentro e fora desse cenário. No entanto, há ações desenvolvidas na intersecção entre a atenção primária e a rede de saúde mental que vão além do sofrimento mental, visto que esse sujeito necessita de cuidados que não cabem na rede especializada, beneficiando-se quando o tratamento é deslocado do local da especialidade para o local que é da vida, do cotidiano das necessidades de saúde ${ }^{(7)}$.

O processo de trabalho dos trabalhadores utiliza diferentes tecnologias para o manejo da demanda dos usuários do sistema de saúde, entretanto, há um ponto de convergência: a relação com o sujeito, produzindo, assim, o que se define como trabalho vivo em ato. São operadores importantes quando se produz saúde em ambientes em que se acolhe doença, adoecimento e saúde também, por meio da promoção à saúde, prevenção de agravos e cuidado( ${ }^{(8)}$.

A relação com o sujeito é algo caro à saúde mental e seu usuário, sendo um operador potente no tratamento, desde a abordagem inicial do sujeito até a manutenção do cuidado. Pensar saúde mental de forma fragmentada não é um bom preditor de ações que beneficiem o sujeito, sua família, rede social e comunidade.

O sofrimento mental não pode ser visto exclusivamente no rol das condições de adoecimento e doença que tenham como horizonte a cura, ele é crônico em sua maioria, grave em alguns casos e se alinha com a capacidade de o sujeito viver independentemente do diagnóstico ou das limitações impostas pela sua condição psíquica.

Nessa perspectiva, é necessário encontrar, nos diferentes pontos de atenção, independentemente da rede onde o tratamento do sujeito em sofrimento mental está inserido, acolhimento e visibilidade. Ser visível e visto aos olhos do trabalhador em saúde faz desse sujeito alguém que reconhecidamente precisa de cuidados em saúde. Desloca o sujeito do local em que, em tese, ele deveria estar, o serviço especializado, para o local onde ele precisa estar naquele momento de sua vida, independentemente da existência de articulação ou não da rede ${ }^{(9)}$.

O presente estudo objetiva apresentar o olhar de duas acadêmicas do último ano da graduação em Enfermagem de uma instituição federal de ensino superior (IFES), durante o internato em um serviço da atenção primária em saúde, em relação ao cuidado em rede à pessoa em sofrimento mental, utilizando essa descrição para discutir o tema com base na literatura.

\section{Método}

O estudo se propõe a relatar a experiência de duas acadêmicas de enfermagem durante o internato de 
quatro meses em um centro de saúde de um município da região metropolitana de Belo Horizonte. A vivência se deu de segunda a sexta-feira durante o horário de funcionamento do centro de saúde, entre os meses de março e junho de 2017, no qual era realizado o acolhimento dos usuários pelas alunas, assim como também o eram outras atividades que compõem a formação do enfermeiro.

Trata-se de um estudo descritivo, do tipo relato de experiência apresentado por meio de uma narrativa das acadêmicas, que foi construída a partir do registro em diários de campo. O projeto de pesquisa foi aprovado sob o número 0482.0.203.000-09 do Comitê de Ética em Pesquisa da Universidade Federal de Minas Gerais ${ }^{(9)}$.

Os resultados são apresentados da seguinte forma: caracterização do município e sua rede de atenção à saúde, com o objetivo de localizar o objeto estudado em tela; a narrativa como um recurso da apresentação do que foi registrado nos diários de campo e a discussão.

\section{Resultados}

\section{A rede de saúde do município}

O centro de saúde era localizado em um município que, segundo dados do Instituto Brasileiro de Geografia e Estatística (IBGE), tinha uma população estimada, em 2017, de 135.968 habitantes e uma área da unidade territorial de 302,419 quilômetros quadrados, ou seja, uma densidade demográfica de 449,6 habitantes por quilômetro quadrado. Em 2010, 97,48\% das pessoas habitavam a área urbana do município. O Índice de Desenvolvimento Humano Municipal (IDHM) em 2010 foi de 0,731 (considerado alto) e o Produto Interno Bruto (PIB) per capita em 2014 foi de R\$ 15.969,67; entretanto, em 2010, 33,2\% da população apresentava rendimento nominal mensal per capita de até meio salário mínimo.

No município existiam, à época da coleta de dados, 22 unidades básicas de saúde e um hospital da rede hospitalar estadual, que realizava cirurgias eletivas por meio dos encaminhamentos advindos da central de regulação. Além disso, possuía uma unidade de pronto atendimento (UPA), uma Santa Casa de Misericórdia, que realizava atendimentos particulares, conveniados, e disponibilizava leitos de internação para o Sistema Único de Saúde, e um centro de atendimento especializado. Em relação ao serviço de saúde mental, ele era composto por dois Centros de Atenção Psicossocial (CAPS), um adulto na modalidade II e outro infantil, para o atendimento da população.

O CAPS II funcionava de segunda a sexta, das 7h às 17h. Era constituído por uma equipe composta por três psiquiatras, dois assistentes sociais, quatro psicólogos, um terapeuta ocupacional, um enfermeiro e três técnicos de enfermagem. $O$ atendimento era realizado por meio de demanda espontânea e encaminhamentos das unidades básicas de saúde, hospitais, secretaria de desenvolvimento social e demais serviços. O usuário era acolhido inicialmente por um profissional da equipe, com formação de nível superior. Após esse primeiro atendimento, o caso era discutido com o restante da equipe e, então, elaborava-se um plano terapêutico.

O serviço oferecia duas modalidades de atendimentos: consultas ambulatoriais e permanência dia. A permanência dia se aplicava a pacientes que estavam em crise e tinham indicação para permanecer no serviço durante todo o horário de funcionamento. Para esses usuários eram oferecidos alimentação e transporte para casa.

O CAPS infantil tinha o funcionamento de segunda a sexta-feira, no horário das $7 \mathrm{~h}$ às $16 \mathrm{~h}$. A equipe era constituída por quatro psicólogos, dois fonoaudiólogos, um terapeuta ocupacional, um médico psiquiatra, um gestor e uma equipe administrativa. Oferecia atendimento às crianças e adolescentes de zero a vinte e cinco anos, encaminhados de escolas, unidades básicas de saúde, conselho tutelar e demanda espontânea. Oferecia consultas com profissionais, além de ter um grupo especializado para autistas. Não possuía permanência dia.

O centro de saúde estava situado em um bairro industrial e residencial, ao lado de uma escola de ensino fundamental e médio e de uma creche, e estava inserido na Estratégia Saúde da Família. Sua população adstrita, segundo dados fornecidos pelo centro de saúde, era dividida em sete microáreas, totalizando $6.026 \mathrm{mil}$ pessoas distribuídas entre regiões urbana e rural, sendo que a área rural estava localizada a, aproximadamente, cinco quilômetros de distância da unidade.

A unidade era pequena e possuía uma equipe de saúde da família, que era composta por: uma enfermeira, que também respondia pela gerência da unidade, duas técnicas de enfermagem, uma médica, um médico de apoio, escalado uma vez por semana na unidade, e sete agentes comunitários de saúde. Os agentes comunitários eram distribuídos em atividades administrativas dentro da unidade, devido à falta de profissionais para realizar tal função. Além disso, a unidade possuía um funcionário da limpeza, um segurança, quatro dentistas e três auxiliares de dentista.

\section{De onde eu te vejo}

Durante o internato em atenção primária, observamos a demanda de pessoas em sofrimento mental, que recorreram ao centro de saúde em busca de atendimento. Em alguns casos, percebemos que as pessoas em sofrimento mental não eram percebidas em sua condição psíquica com facilidade; outras vezes, 
eram rotuladas ou estigmatizadas como pacientes "problemáticos" ou "pacientes CAPS".

A nossa percepção era de que o vínculo entre a atenção primária e a atenção secundária mostrava-se inexistente, ou seja, o que era para ser uma rede de atenção interligada que proporciona autonomia e segurança à pessoa em sofrimento mental não funcionava como deveria. Em alguns casos, observamos que o vínculo com o CAPS era interrompido ou consideravelmente espaçado, e o usuário do serviço passava a integrar e frequentar somente o centro de saúde - na maioria das vezes para renovar uma receita ou com o objetivo de minimizar o ócio em que se encontrava.

Nas observações do cotidiano do serviço identificamos, no nosso ponto de vista, pontos de fragilidade relevantes em relação ao CAPS do município: a dificuldade de atendimento à demanda, o horário de atendimento reduzido, o não atendimento às necessidades de saúde da população, considerando o seu funcionamento limitado quando comparamos com a demanda que se apresentava no centro de saúde.

Além disso, tem-se o número de vagas para atendimento em relação à demanda, como o tempo de espera de quatro a seis meses para atendimento no CAPS, o fato de as consultas serem realizadas exclusivamente com profissional psiquiatra e a falta constante do fornecimento de alguns medicamentos pelo serviço.

Em relação ao centro de saúde, percebe-se a fragilidade na capacitação da equipe de saúde da atenção primária para a recepção e acompanhamento da pessoa em sofrimento mental em suas demandas diárias. Os encaminhamentos do serviço especializado para o centro de saúde eram percebidos como uma via de mão única onde a lógica era 'desafogar' o CAPS em relação ao número de atendimentos, sem considerar a formação dos profissionais e suas dificuldades para o manejo das pessoas em sofrimento mental.

Percebemos a vulnerabilidade dos usuários, além da complexidade por parte do profissional no manejo desses sujeitos, e as dificuldades para tratar e cuidar de uma pessoa em sofrimento mental. Foi observada a presença de outras comorbidades clínicas, além do transtorno mental, em especial diabetes e hipertensão, sendo um dos principais desafios da equipe articular as necessidades e demandas desse usuário do serviço com a capacidade de manejo nestes casos.

Para os profissionais do centro de saúde, percebe-se tratar-se de um desafio realizar ações educativas de prevenção de agravos e promoção da saúde para essa clientela, assim como cuidar das comorbidades associadas.

Ao final das observações, em discussão com a docente orientadora, percebe-se um olhar da realidade sem articular a prática com a teoria, desprovido de articulações necessárias para compreender a realidade.
A partir dessa percepção, surge a necessidade de buscar na literatura elementos para compreender o vivido sob outra perspectiva, que enriquecesse a formação e afastasse do senso comum.

\section{Discussão}

O internato no último ano da graduação em enfermagem é um momento importante para a formação do futuro enfermeiro, em que se espera que as perguntas sobre o trabalho do enfermeiro nos serviços de saúde sejam respondidas e onde se fazem e elaboram novos questionamentos sobre a teoria e a prática, sobre a realidade do fazer e do saber-fazer em saúde, em especial onde o estudante faz uma aproximação da realidade vivida pelo trabalhador da saúde - enfermeiro - nas diferentes redes de atenção à saúde(10).

A literatura aponta que o ensino de enfermagem psiquiátrica está pautado em formas tradicionais de construir conhecimento com e para o aluno, mas que muitos cursos realizam movimentos, com a utilização de metodologias ativas, para que os futuros enfermeiros possam apreender que o sofrimento mental tem suas particularidades, sendo uma condição crônica de adoecimento que não pode ser compreendido como única necessidade de saúde do indivíduo, em detrimento de outras condições de adoecimento, quer sejam agudas ou crônicas ${ }^{(11-12)}$.

Um dos desafios da formação do enfermeiro nesse sentido é agregar aos currículos conteúdos que considerem as questões biológicas dos transtornos, mas que não deixem de lado as questões psicossociais e os determinantes sociais de saúde e de saúde mental. Esse equilíbrio pode proporcionar ao futuro enfermeiro um olhar ampliado para a realidade de um serviço de saúde, como esta descrita no presente artigo, considerando, por exemplo, a população do município, sua densidade demográfica, a ocupação do espaço urbano, o IDHM, PIB e a renda nominal per capita, assim como a rede de saúde possível para esse município, considerando sua arrecadação e gestão do que é arrecadado, entre outros fatores.

Quando se tem essa compreensão, é possível olhar para o serviço de outra forma, como um espaço que reflete a gestão da saúde no munícipio, as relações entre os serviços, como se organiza a rede, a relação dos profissionais com o sujeito em sofrimento mental, as condições de trabalho dos trabalhadores dos serviços que atendem à mesma demanda e têm pontos de convergência e divergência, o estranhamento causado pelo cuidado e não cuidado oferecido para a necessidade de saúde daquele sujeito, que pode ser realizado na atenção primária ou secundária, dependendo da demanda que ele apresenta.

O estigma social que a pessoa em sofrimento mental carrega está descrito na literatura e faz parte 
da vida desse sujeito, assim como promover a não estigmatização faz parte da rotina da rede de saúde mental e deveria estar presente na agenda dos demais serviços de saúde. O estigma gera a invisibilidade do sujeito e da sua demanda, que passa a ser classificada como única. Além do diagnóstico, ao sujeito é imputado pertencer a um determinado local: o serviço especializado, no caso, o CAPS. Nesse caso ao sujeito resta o incômodo que causa ao serviço, por não saber o que fazer com ele e com sua demanda(13).

Para além dessa questão, perde-se naquele momento a oportunidade de transformar o cuidado integral em ato social, político e de saúde. o campo de estudos da Saúde Mental Global contribui para essa compreensão, pois ao discutir formas de reduzir as iniquidades no acesso e no cuidado destinado a essa população, aponta três estratégias que podem contribuir de forma direta e indireta para essa questão, a saber: a integração dos serviços de saúde mental aos serviços de atenção primária à saúde, o compartilhamento e delegação de tarefas e a incorporação de inovações tecnológicas nos modelos de ofertas de serviços existentes de saúde mental(14)

Cabe lembrar que, em 2020, segundo dados da Organização Mundial de Saúde, a depressão será a segunda maior causa de incapacidade no mundo. Os serviços de saúde mental não terão capacidade para o manejo de tantos $\operatorname{casos}^{(15)}$.

A integração dos serviços no caso brasileiro trabalha com a referência e contrarreferência, com o apoio matricial e a articulação das redes. Sua operacionalização apresenta nós que contam com a inventividade dos atores envolvidos e implicados para solucionar as dificuldades do dia a dia das ações em conjunto. O compartilhamento e delegação de tarefas pode ser apreendido no contexto do funcionamento do trabalho em rede como a estratégia virtuosa do matriciamento em saúde mental, mecanismo poderoso para desvencilhar casos complexos que necessitam de diferentes abordagens, envolvimento de atores e instituições diversas no manejo dentro e fora do território(16).

O compartilhamento e delegação de tarefas, assim como a transferência de tecnologias do cuidado operadas dentro do serviço de saúde mental, nos parecem uma via importante que merece investimento na produção de cuidado da pessoa em sofrimento mental na atenção básica. Não é suficiente olhar para o CAPS naquilo que o olhar vê como fragilidade; cabe reflexão sobre o potencial de criatividade e inventividade desse serviço na produção de autonomia do sujeito, de viabilidade de projetos pessoais e coletivos e na forma como isso é gestado dentro do serviço de saúde mental para se dar no cotidiano do território.
O compartilhamento desse saber-fazer pela equipe do CAPS com as equipes de saúde da atenção primária pode gerar ações mais fluidas no cuidado do outro, um outro que além do sofrimento mental, convive com comorbidades de outras clínicas, que em alguns casos, põem em risco a sua saúde e precisam ser abordadas.

A educação permanente é o ponto que sustenta a oferta de serviços de qualidade pelo trabalhador de saúde independentemente da rede de cuidados na qual ele se insere. Para produzir cuidado qualificado, ético e estético em saúde, é necessário que o trabalhador esteja seguro de suas ações, tenha apoio e suporte da equipe e do serviço enquanto componente de uma rede. As necessidades de saúde do sujeito que procura a atenção primária são as mais diversas, portanto, os profissionais precisam ser capacitados para lidarem com essa pluralidade, ao mesmo tempo em que não podem perder o horizonte do que é de cada um em sua singularidade(17).

É um equilíbrio complexo e que demanda esforço por parte da gestão do sistema de saúde como um todo, em todos os seus níveis. O manejo do usuário da saúde mental tem particularidades em alguns momentos, entretanto, em outros, ele não difere do que um usuário de outra clínica necessita no que concerne à sua saúde. Podemos inferir que essa dificuldade está associada à ideia de periculosidade, ao estigma e preconceito, ao medo, o que nos levaria a uma discussão histórica, política e social sobre o sofrimento mental. Outro caminho é pensar que essas questões podem ser minimizadas se apreendidas como desafios do cuidado do território e de seus entes ${ }^{(18)}$.

A literatura, como ponto de apoio para a compreensão do que foi visto, gerou a possibilidade de discussão junto às alunas da necessidade da articulação entre teoria e prática, partindo do princípio de que o olhar do profissional de saúde não pode ser deslocado desse horizonte.

Compreende-se que existam nós nos pontos de convergência e divergência das diferentes redes que compõem a atenção em saúde no que concerne a pessoa em sofrimento mental, mas para além dessas discussões necessárias, o olhar/texto, nesse caso, foi um pretexto para discutir alguns nós existentes no campo de encontros e desencontros da rede e serviços de saúde mental com a atenção primária em saúde, tendo como pano de fundo a formação do aluno de graduação.

\section{Considerações Finais}

O estudo apresenta os limites próprios de um relato de experiência. O primeiro é que não existe possibilidade de generalizações. Segue-se a este a visão de duas alunas sobre determinado tema associado a um contexto específico onde se devem considerar elementos não 
só do cenário de observação, mas também do próprio sujeito que observa. Daí a necessidade de realização de outros estudos, com diferentes abordagens teóricas que explorem a temática do olhar do futuro profissional enfermeiro em relação aos serviços de saúde onde se dá a sua formação.

A presente discussão partiu do olhar do enfermeiro em formação sobre uma questão complexa. Ela buscou, junto à literatura sobre o tema, contribuir para a discussão sobre as necessidades de cuidado das pessoas em sofrimento mental e como isso é percebido pelo estudante de enfermagem, ancorado em conceitos e pressupostos teóricos que têm por objetivo contribuir para pensar saúde de forma articulada, considerando seus determinantes sociais, políticos e financeiros, entre outros, no que concerne à formação do enfermeiro.

Conhecer o ponto de vista do aluno nesse sentido contribui para pensar a prática docente, rever conteúdos e formas de abordá-los, pensar nas fragilidades do que foi aprendido, mas não necessariamente foi apreendido. É necessário articular discussões que considerem a realidade dos serviços e considerar o local onde eles se inserem torna-se um importante ponto no planejamento da formação em saúde mental e em saúde quando se pensa no trabalho em redes dentro de um sistema que é único.

\section{Referências}

1. Amarante $P$, Nunes $M O$. A reforma psiquiátrica no SUS e a luta por uma sociedade sem manicômios. Ciênc Saúde Coletiva. [Internet]. junho 2018 [Acesso 14 ago 2019]; 23(6):2067-74. Disponível em: http://www. scielo.br/pdf/csc/v23n6/1413-8123-csc-23-06-2067.pdf 2. Frosi RV, Tesser Charles D. Mental health care practices in primary health care: an analysis based on experiences developed in Florianópolis, Brazil. Ciênc Saúde Coletiva. [Internet]. 2015 Oct [cited 2019 Aug 14]; 20(10):3151-61. Available from: http://www.scielo. br/pdf/csc/v20n10/en_1413-8123-csc-20-10-3151.pdf 3. Moliner J, Lopes SMB. Saúde mental na atenção básica: possibilidades para uma prática voltada para a ampliação e integralidade da saúde mental. Saúde Soc. [Internet]. $2013 \mathrm{Dec}$ [Acesso 14 ago 2019]; 22(4):1072-83. Disponível em: http://www.scielo.br/ pdf/sausoc/v22n4/10.pdf

4. Adler NE, Glymour MM, Fielding J. Addressing Social Determinants of Health and Health Inequalities. JAMA. [Internet]. 2016 Dec [cited 2019 Aug 14]. 316(16):1641-2. Available from: https://jamanetwork. com/journals/jama/article-abstract/2556011

5. Silva M, Loureiro A, Cardoso G. Social determinants of mental health: a review of the evidence. Eur J Psychiat. [Internet]. 2016 Dec [cited 2019 Ago 14]; 30(4):259-92. Disponível em: http://scielo.isciii.es/pdf/ ejpen/v30n4/original03.pdf
6. Paim JS. Sistema Único de Saúde (SUS) aos 30 anos. Ciênc Saúde Coletiva. [Internet]. 2018 June [Acesso 14 ago 2019]; 23(6):1723-8. Disponível em: http://www. scielo.br/pdf/csc/v23n6/1413-8123-csc-23-06-1723.pdf 7. Hirdes A, Scarparo HBK. O labirinto e o minotauro: saúde mental na Atenção Primária à Saúde. Ciênc Saúde Coletiva. [Internet]. 2015 Feb [Acesso 14 ago 2019]; 20(2):383-93. Disponível em: http://www.scielo.br/pdf/ csc/v20n2/1413-8123-csc-20-02-0383.pdf

8. Santos DS, Mishima SM, Merhy EE. Processo de trabalho na Estratégia de Saúde da Família: potencialidades da subjetividade do cuidado para reconfiguração do modelo de atenção. Ciênc Saúde Coletiva. [online]. 2018 [Acesso 14 ago 2019]; 23(3):861-70. Disponível em: http://www.scielo.br/pdf/ csc/v23n3/1413-8123-csc-23-03-0861.pdf

9. Minayo MCS. O desafio do conhecimento: pesquisa qualitativa em saúde. 14. ed. São Paulo: Hucitec; 2014. 10. Lana FCF. Projeto "Ações integradoras no âmbito da atenção primária à saúde vinculadas ao estágio curricular I - EEUFMG". [tese]. Belo Horizonte: Escola de Enfermagem da Universidade Federal de Minas Gerais; 2019.

11. Rodrigues WO, Mourão LC, Almeida ACV, Oliveira GS. Os limites do ensino teórico-prático da saúde mental na formação do profissional de saúde. Rev Portuguesa Enferm Saúde Mental. [Internet]. out 2016 [Acesso 14 ago 2019]; (spe4): 107-14. Disponível em: http://www. scielo.mec.pt/pdf/rpesm/nspe4/nspe4a16.pdf

12. Costal CRB, Oliveira LB, Maynart WHC, Albuquerque MCS, Correia DS. Perceptions of the nursing students on stress-generating factors during the graduation. Rev Rene. 2018; [cited 2019 Aug 14] 19:e3442. Available from: http://periodicos.ufc.br/rene/article/view/33471 13. Klika AK, Williamsb SL, Reynoldsa KJ. Toward understanding mental illness stigma and help-seeking: A social identity perspective. Soc Scienc Medicine. 2019 Feb [cited 2019 Aug 14]. 222: 35-43. Available from: https://www.sciencedirect.com/science/article/pii/ S0277953618306749?via\%3Dihub

14. Wenceslau LD, Ortega F. Saúde mental na atenção primária e Saúde Mental Global: perspectivas internacionais e cenário brasileiro. Interface. (Botucatu) [Internet]. 2015 Dec [cited 2019 Aug 14]; 19(55):1121-32. Available from: http://www.scielo.br/ pdf/icse/v19n55/1807-5762-icse-1807-576220141152.pdf 15. World Health Organization. Investing in mental health: evidence for action. Geneva: WHO; 2013. [cited 2019 Aug 14] Available from: https://apps.who.int/iris/ handle/10665/87232

16. Ministério da Saúde (BR). Secretaria de Atenção à Saúde. Departamento de Ações Programáticas Estratégicas. Brasília: Ministério da Saúde; 2015. 548 p. (Caderno Humaniza SUS; v. 5) 
17. Marcolino TQ, Fantinatti EN, Gozzi APNF. Comunidade de prática e cuidado em saúde mental: uma revisão sistemática. Trabalho Educ Saúde. [Internet]. 2018 [Acesso 14 ago 2019]; 16(2):643-58. Disponível em: http://www.scielo.br/pdf/tes/v16n2/1678-1007-tes1981-7746-sol00112.pdf

18. Barbosa VFB, Caponi SN, Verdi MIM. Risco como perigo persistente e cuidado em saúde mental: sanções normalizadoras à circulação no território. Saúde Soc. [Internet]. 2018 [Acesso 14 ago 2019]; 27(1):175-84. Disponível em: http://www.scielo.br/pdf/sausoc/ v27n1/1984-0470-sausoc-27-01-175.pdf

\section{Contribuição dos autores}

Concepção e desenho da pesquisa: Amanda Márcia dos Santos Reinaldo. Obtenção de dados: Bárbara Jéssica de Melo Cezar Dias e Fernanda Martinez de Oliveira. Análise e interpretação dos dados: Amanda Márcia dos Santos Reinaldo, Bárbara Jéssica de Melo Cezar Dias e Fernanda Martinez de Oliveira. Obtenção de financiamento: sem financiamento. Redação do manuscrito: Bárbara Jéssica de Melo Cezar Dias, Fernanda Martinez de Oliveira Resende, Giulia Ribeiro Schettino Regne, Amanda Márcia dos Santos Reinaldo, Maria Odete Pereira e Marcus Luciano de Oliveira Tavares. Revisão crítica do manuscrito quanto ao conteúdo intelectual importante: Amanda Márcia dos Santos Reinaldo.

Todos os autores aprovaram a versão final do texto.

Conflito de interesse: os autores declararam que não há conflito de interesse.
Recebido: 23.01.2019

Aceito: 31.01 .2020 